\title{
Effect of stacking sequence on properties of coconut leaf sheath/jute/E-glass reinforced phenol formaldehydehybrid composites
}

\begin{abstract}
In the present study, the effect of stacking sequence on mechanical, physical, and biodegradability properties of treated coconut leaf sheath/jute/glass fabric reinforced phenol formaldehyde hybrid composites were studied. The hybrid composite laminates were fabricated by using hand lay-up technique. The specimen preparation and testing were conducted as per ASTM standards. Obtained results indicated that the incorporation of coconut leaf sheath with glass fiber of hybrid composites has greater effect on tensile and hardness properties. Coconut leaf sheath fibers treated with $\mathrm{NaOH}$ solution shows significant improvement for adhesion between fiber and matrix. Water absorption plots for different periods of immersion indicated that coconut leaf sheath with glass hybrid composites offer better resistance to water absorption than pure natural fiber reinforced composites. Thickness swelling of the hybrid composites increases due to increase in the percentage of water absorption of the composites. From SEM analysis of the tensile fractured surface of hybrid composites, it is found that the failure of composite is due to poor interfacial bonding between fiber and matrix. An overall comparison of all the laminates revealed that the hybrid laminates of coconut leaf sheath with extreme glass fibers plies as skin layer is the optimum combination with a good balance between the properties. So they may be suited in manufacturing of car doors, car interiors, dash boards, headliners, decking, parcel shelves, pallets, spare tyre covers, spare-wheel pan, seat backs, etc.
\end{abstract}

Keyword: Coconut leaf sheath; Jute; E-glass; Phenol formaldehyde; Hand lay-up; Hybrid composites 
